\title{
Association of GSTM1 and GSTT1 Null Genotypes with Toluene Diisocyanate-Induced Asthma
}

\author{
Jong-Uk Lee $\mathbb{D}^{\circ},{ }^{1}$ Ji-Yeon Jeong $\mathbb{D}^{1},{ }^{1}$ Min Kyung Kim $\mathbb{D}^{,},{ }^{2}$ Sun A. Min, ${ }^{2}$ Jong-Sook Park $\mathbb{D}^{1}$, \\ and Choon-Sik Park $\mathbb{D}^{1,3}$ \\ ${ }^{1}$ Division of Allergy and Respiratory Medicine, Department of Internal Medicine, Soonchunhyang University Bucheon Hospital, \\ Bucheon, Republic of Korea \\ ${ }^{2}$ Department of Interdisciplinary Program in Biomedical Science Major, Soonchuhyang University, Asan, Republic of Korea \\ ${ }^{3}$ PulmoBioPark Co., Ltd., Soonchunhyang University Bucheon Hospital, Bucheon, Republic of Korea
}

Correspondence should be addressed to Jong-Sook Park; newstart1221@naver.com

Received 26 August 2021; Accepted 15 January 2022; Published 11 February 2022

Academic Editor: Michael Roth

Copyright $\odot 2022$ Jong-Uk Lee et al. This is an open access article distributed under the Creative Commons Attribution License, which permits unrestricted use, distribution, and reproduction in any medium, provided the original work is properly cited.

\begin{abstract}
Background. Toluene diisocyanate (TDI) causes occupational asthma by generating oxidative stress, leading to tissue injury and inflammation. Glutathione transferases (GSTs) are detoxifying enzymes that eliminate oxidative stress. We examined whether the genotypes of the GSTM1 and GSTT1 genes are associated with TDI-induced occupational asthma (TDI-OA). Methods. The study population consisted of 26 asthmatics with a positive response to the TDI challenge (TDI-PA) and 27 asthmatics with negative responses (TDI-NA). GSTM1 and GSTT1 null and wild-type genotypes were determined using multiplex PCR. The plasma GSTM1 and GSTT1 protein concentrations were determined using ELISA. Results. The GSTM1 null genotype was more frequent in the TDI-PA than in the TDI-NA ( $77.8 v s .50 .0 \%, \mathrm{OR}=3.5, p=0.03)$, while the frequency of the GSTT1 null genotype tended to be higher in the TDI-PA than in the TDI-NA (59.3 vs. $42.3 \%$, OR $=1.98, p=0.21)$. When analyzed together, the GSTM1/GSTT1 null genotype was more frequent in the TDI-PA than in the TDI-NA ( $48.2 v s .15 .3 \%, \mathrm{OR}=6.5, p=0.04)$. The decline in the FEV in $1 \mathrm{~s}$ after TDI challenge was higher with the GSTM1/GSTT1 null than the GSTM1 wild-type/GSTT1 null genotypes (24.29\% vs. $7.47 \%, p=0.02)$. The plasma GSTM1 level was lower with the GSTM1 null than with the GSTM1 wild-type genotypes both before $(13.7 v s .16 .6 \mathrm{ng} / \mathrm{mg}, p=0.04)$ and after $(12.9 v s .17 .1 \mathrm{ng} / \mathrm{mg}$, $p=0.007)$ the TDI challenge, while the GSTT1 level was not changed with either the GSTT1 null or wild-type genotype. Conclusions. The GSTM1 null genotype, but not GSTT1 alone, may confer susceptibility to TDI-OA. However, the genetic effect of the GSTM1 null genotype may be enhanced synergistically by the GSTT1 null genotype. The genetic effect of GSTM1 was validated in the plasma as the GSTM1 protein level. Therefore, the GSTM1 and GSTT1 genotypes may be useful diagnostic markers for TDI-OA.
\end{abstract}

\section{Introduction}

Highly reactive toluene diisocyanate (TDI), diphenylmethane diisocyanate, and hexamethylene diisocyanate are widely used globally for manufacturing polyurethane foams, paints, and lacquers and cause occupational asthma (OA) [1] accounting for more than half of the OA cases in Korea [2]. TDI-induced OA (TDI-OA) is characterized by airway hyper-responsiveness, inflammation, and remodeling $[3,4]$. Several immunological and nonimmunological mechanisms are thought to be involved in the pathogenesis of TDI-OA, as in other types of OA $[5,6]$. One nonimmunological mechanism involves physical interactions between diisocyanate and airway epithelium that induce the production of proinflammatory cytokines and chemokines, which recruit inflammatory cells [7]. Isocyanates also induce oxidative stress directly in inflammatory monocyte cell lines [8], lymphocytes [9], neutrophils [10], constitutional epithelial [11], and endothelial [12] cell lines. These processes are accompanied by redox imbalances that overwhelm antioxidant systems in the airways [13] as well as systemically [14]. Several complex antioxidant systems protect cells from oxidative stress, such as glutathione S-transferases (GSTs), thioredoxin peroxidases, catalases, glutathione reductases, and superoxide dismutases [15]. 
GSTs are a family of eukaryotic and prokaryotic phase II metabolic isozymes that catalyze the conjugation of the reduced form of glutathione to xenobiotic substrates for detoxification [16]. The GST family includes cytosolic, mitochondrial, and microsomal proteins [17]. Human cytosolic GSTs can be divided into five main classes: alpha, mu, pi, theta, and zeta [18]. The human GSTM (GST $\mu$ ) gene family on chromosome 1p13.3 consists of five different isotypes, GSTM1 to GSTM5 [19], and the theta class (GSTT) on chr22 includes GSTT1, GSTT2, and GSTT2B [20]. The null genotypes result in no GST function, and therefore the conjugation reaction and subsequent elimination of the toxic products of oxidative stress are inefficient, which causes inflammatory and immune dysfunction in chronic disorders. GSTM1 mutations are associated with risks of inflammatory bowel diseases [21], type 2 diabetes mellitus [22], and atherosclerotic cardiovascular diseases [23]. GSTM1 and GSTT1 null genotypes are also associated with risks of chronic obstructive lung disease [24] and lung cancer [25].

A recent meta-analysis demonstrated that GSTM1 and GSTT1 null genotypes are associated with an increased risk of asthma [26]. TDI-OA may develop more frequently in individuals with null genotypes than in those with wild-type genotype via enhanced oxidative stress in the airways and whole body[14]. Isocyanate-induced asthma is associated with the GSTM1 null genotype in Caucasians [27], and the GSTP1 Val/Val genotype is less frequent in asthmatics exposed to TDI for 10 or more years [28]. Thus, individuals with the null genotypes for both GSTM1 and GSTT1 may be susceptible to TDI-OA in a synergistic manner. However, few studies have examined the association between the GSTM1/GSTT1 null genotypes and TDI-OA. Furthermore, most studies diagnosed TDI-OA based on a history of work exposure to the causative agent and the presence of asthma symptoms. However, this approach is not always satisfactory for diagnosing OA [29]. Thus, in the present study, TDI-OA was diagnosed by specific inhalation challenge (SIC) using TDI solution because SIC is considered the standard reference test [30]. Then, the distributions of deletion mutants of GSTM1 and GSTT1 were compared between TDI-challenge-positive (TDI-PA) and -negative (TDI-NA) asthma patients to explore the possible associations between the null genotypes and the risk for TDI-OA. Concomitantly, the plasma GSTM1 and GSTT1 protein concentrations were analyzed to validate the genetic effects.

\section{Materials and Methods}

2.1. Study Subjects. Buffy coats and plasma $(n=53)$ were obtained from a biobank at Soonchunhyang University Hospital, Bucheon (schbc-biobank-2019-009-01), Korea, after the study was approved by the Ethics Committee of Soonchunhyang University (IRB No. 201905-BR-020-01). Informed written consent for study participation and sample donation was obtained from each subject. Additionally, this study used fully anonymized data collected previously as part of the biobank at Soonchunhyang University Bucheon Hospital.
All subjects had a history of work exposure and asthma symptoms; however, 27 subjects had positive (TDI-PA group) and 26 negative (TDI-NA group) responses to SIC with TDI, as described previously [31]. The decline in the forced expiratory volume in $1 \mathrm{~s}$ (FEV1) after the TDI challenge (dFEV1-TDI) was calculated as ((FEV1 before challenge - the lowest FEV1 after provocation)/FEV1 before challenge $) \times 100(\%)$. Atopy was determined by a positive skin test to at least one of the following common inhalant allergens: house dust mites, tree, grass, weed pollens, animal dander, and Alternaria (Bencard, Brentford, UK).

2.2. Isolation of DNA and Genotyping of GSTM1 and GSTT1. DNA was isolated using the QIAGEN DNA isolation kit following the manufacturer's protocol (QIAGEN, Hilden, Germany). The DNA concentration and quality were measured using the NanoDrop instrument (NanoDrop Technologies, Wilmington, DE, USA) and by agarose gel electrophoresis. The GSTM1 and GSTT1 genotypes were determined simultaneously in a single multiplex PCR assay (Figure 1(a)). Briefly, isolated DNA (20 ng) was amplified in a $25 \mu \mathrm{L}$ reaction mixture containing $10 \mathrm{pmol}$ GSTM1 ( $5^{\prime}$ GAACTCCCTGAAAAGCTAAAGC and $5^{\prime}$ GTTGGGCTCAAATATACGGTGG) and GSTT1 (5' TICCTTACTGGTCCTCACATCTC and 5'-TCACCGGATCATGGCCAGCA) primers. As an internal control, albumin was coamplified using the primers $5^{\prime}$ GCCCTCTGCTAACAAGTCCTAC and $5^{\prime}$ GCCCTAAAAAGAAAATCGCCAATC. PCR was performed using the Multiplex PCR assay kit (Takara Bio, Shiga, Japan) under the following protocol: $5 \mathrm{~min}$ at $94^{\circ} \mathrm{C}$, followed by 30 cycles at $94^{\circ} \mathrm{C}$ for $1 \mathrm{~min}, 63^{\circ} \mathrm{C}$ for $1 \mathrm{~min}$, and $72^{\circ} \mathrm{C}$ for $1 \mathrm{~min}$. The PCR products were analyzed electrophoretically on ethidium bromide-stained $1.5 \%$ agarose gels. The absence of GSTM1 or GSTT1 PCR products was defined as the null genotype (Figure 1(b)).

\subsection{Measuring Plasma GSTM1 and GSTT1 Protein} Concentrations. The plasma GSTM1 and GSTT1 concentrations were measured using an ELISA kit (MyBioSource, San Diego, CA, USA) according to the manufacturer's instructions. A microtiter plate was precoated with antibodies specific for GSTM1 and GSTT1. The absorbance at $450 \mathrm{~nm}$ was determined by an ELISA reader, and the GSTM1 and GSTT1 protein concentrations were determined by the optical density of the samples in comparison with a standard curve. The total protein concentration was determined by the BCA assay using bovine serum albumin as a standard.

2.4. Statistical Analysis. The data were analyzed using IBM SPSS ver. 20.0. (IBM, Armonk, NY, USA). The Pearson $\chi^{2}$ test was used to compare the differences in the GSTM1 and GSTT1 genotype frequencies between TDI-PA and TDI-NA. The relative risks of the genotypes for TDI-PA were analyzed using logistic regression and presented using odd ratios (ORs) with 95\% confidence intervals (CIs). The GSTM1 and GSTT1 protein concentrations were compared between 


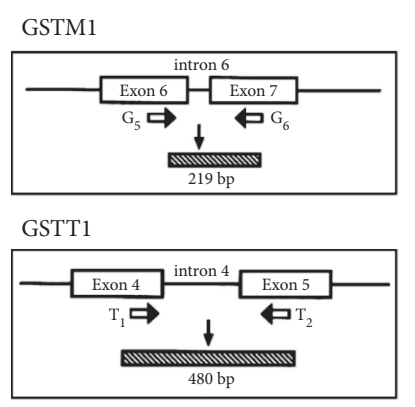

(a)

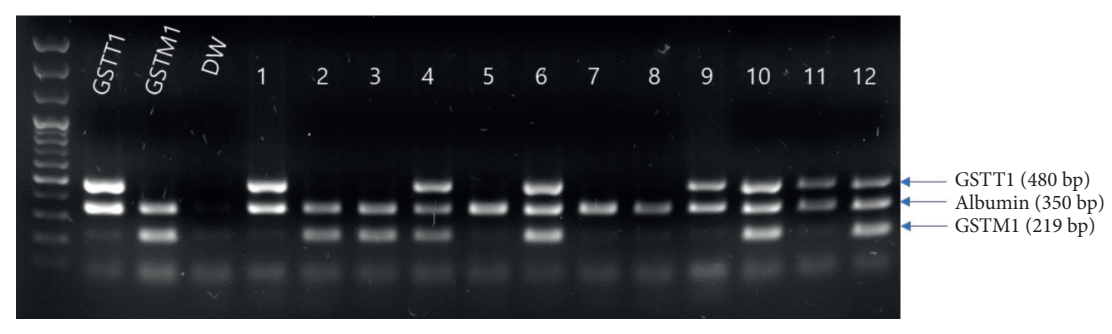

(b)

FIGURE 1: Primer positions (a) and gel electrophoresis of GSTM1 and GSTT1 RT-PCR products and albumin (b). Lanes 1, 9, and 11: GSTT1 wild/GSTM1 null genotype; lanes 5, 7, and 8: GSTT1/GSTM1 null genotype; lanes 4, 6, 10, and 12: GSTT1/GSTM1 wild genotype; and lanes 2 and 3, GSTT1 null/GSTM1 wild genotype.

TDI-PA and TDI-NA according to the GST genotype using the nonparametric Mann-Whitney $U$-test and the Wilcoxon signed rank test after testing for normality using the Shapiro-Wilk test. Values of $p<0.05$ were considered significant.

\section{Results}

3.1. Comparing GSTM1 and GSTT1 Null Genotypes between the TDI-PA and TDI-NA Groups. Of the 53 asthmatics, 27 were TDI-PA (51\%) and 26 were TDI-NA (49\%). The TDIPA group had a significantly greater FEV1 decline after the TDI challenge and a higher serum total IgE level compared with those of the TDI-NA group $(p<0.05)$ (Table 1). Table 2 shows the distributions of GSTM1 and GSTT1 null genotypes. The respective frequencies of the GSTM1 null and wild-type genotypes were $77.8 \%$ and $22.2 \%$ in the TDI-PA group $(n=27)$ and $50.0 \%$ and $50.0 \%$ in the TDI-NA group $(n=26)$ (Table 2$)$. The proportion of participants with the null genotype was significantly higher in the TDI-PA group (OR: 3.5, 95\% CI: 1.06-11.49, $p=0.035$ ) (Table 2). The respective frequencies of the GSTT1 null and wild-type genotypes were $59.3 \%$ and $40.7 \%$ in the TDI-PA group and $42.3 \%$ and $57.7 \%$ in the TDI-NA group (Table 2 ). The proportion of participants with the null genotype tended to be higher, but not significantly, in the TDI-PA group $(p=0.217)$.

3.2. Combined Effect of GSTM1 and GSTT1 Null Genotypes on the TDI-Induced Response. The study subjects were divided into four groups according to the wild-type and null genotypes of both GSTM1 and GSTT1: (1) GSTM1/GSTT1 wild; (2) GSTM1 null/GSTT1 wild; (3) GSTM1 wild/GSTT1 null; and (4) GSTM1/GSTT1 null. In the TDI-PA group $(n=27)$, GSTM1/GSTT1 null was the most frequent genotype $(n=13,48.2 \%)$, followed by GSTM1 null/GSTT1 wild $(n=8,29.6 \%)$, GSTM1 wild/GSTT1 null $(n=3,11.1 \%)$, and GSTM1/GSTT1 wild ( $n=3,11.1 \%)$ (Table 3$)$. In the TDI-NA group $(n=26)$, GSTM1 null/GSTT1 wild was the most frequent genotype $(n=9,34.6 \%)$, followed by GSTM1 wild/ GSTT1 null $(n=7,26.9 \%)$, GSTM1/GSTT1 wild $(n=6$, $23.0 \%)$, and GSTM1/GSTT1 null $(n=4,15.3 \%)$. Accordingly, the GSTM1/GSTT1 null genotype was three times more frequent in the TDI-PA than in the TDI-NA groups $(\mathrm{OR}=6.50$ (95\% CI: 1.09-38.63), $p=0.04)$.

The dFEV1-TDI $(($ pre - post FEV1)/pre FEV1 1 100)) was evaluated according to the GSTM1 and GSTT1 genotypes: dFEV1-TDI tended to be higher with the GSTM1 null than wild-type genotypes $(21.17 \%$ vs. $7.38 \%, p=0.06)$ (Figure 2(a)) and with the GSTT1 null than wild-type genotypes $(20.39 \%$ vs. $7.45 \%, p=0.1$ ) (Figure $2(\mathrm{~b})$ ). In a combined analysis of the GSTM1 and GSTT1 genotypes, dFEV1-TDI was highest with the GSTM1/GSTT1 null type among the four genotypes, with a significant difference compared with the GSTM1 wild/GSTT1 null genotype (24.29\% vs. $7.47 \%, p=0.029)$ (Figure 2(c)).

3.3. GSTM1 and GSTT1 Protein Levels in Plasma according to Genotype. The plasma GSTM1 protein concentrations did not change after the TDI challenge in the null $(n=31)$ or wild-type $(n=12)$ GSTM1 genotypes (Figure 3(a)). However, the concentrations were significantly lower with the GSTM1 null than with the wild-type genotype before (13.7 vs. $16.1 \mathrm{ng} / \mathrm{mg}$ protein, $p=0.04)$ and after (12.9 vs. $17.1 \mathrm{ng} /$ mg protein, $p=0.007$ ) (Figure $3(\mathrm{a})$ ) the TDI challenge. The plasma GSTT1 protein concentration did not change after the TDI challenge with the null $(n=25)$ or wild-type $(n=18)$ GSTT1 genotype (Figure 3(b)). The GSTT1 protein concentration did not differ between GSTT1 null and wild-type before and after the TDI challenge (Figure 3(b)).

\section{Discussion}

We found that the GSTM1 null genotype was more frequent in the TDI-PA than in the TDI-NA group and was associated with a three-fold increased risk of developing asthma after TDI exposure. In comparison, the frequency of the GSTT1 null genotype was increased, but not significantly, in the TDI-PA group. This suggests that the GSTM1 null genotype, rather than the GSTT1 null genotype, has a dominant genetic effect on susceptibility to TDI-OA. Because GSTM1 and GSTT1 are involved in conjugation reactions and elimination of toxins to produce oxidative stress and subsequent airway inflammation [32, 33], reduced GST enzymatic 
TABLE 1: Clinical characteristics of the study subjects.

\begin{tabular}{lccc}
\hline Variables & TDI-PA & TDI-NA & $P$ value \\
\hline$N$ & 27 & 26 & - \\
Age (years) & $58.7 \pm 8.6$ & $57.1 \pm 10.9$ & $21 / 5$ \\
Sex (male, female) & $23 / 4$ & $6 / 9 / 11$ & 0.60 \\
Smoke (NS/ES/SM) & $9 / 12 / 6$ & $15 / 11$ & 0.72 \\
Atopy (Y, N) & $19 / 8$ & $92.96 \pm 12.62$ & 0.39 \\
FVC (\% predicted) & $87.48 \pm 21.31$ & $97 \pm 13.07$ & 0.26 \\
FEV1 (\% predicted) & $90.22 \pm 24.86$ & $81.42 \pm 9.06$ & 0.22 \\
FEV1_FVC (\%) & $76.22 \pm 16.67$ & $4.57 \pm 3.98$ & 0.16 \\
Decline of FEV1 after the TDI challenge (\%) & $35.15 \pm 15.13$ & $154.23 \pm 200.42$ & $3.66 E-11$ \\
Serum total IgE (kU/I) & $463.18 \pm 689.94$ & 0.03 \\
\hline
\end{tabular}

Numeric data are presented as mean \pm standard deviation. TDI, toluene diisocyanate; TDI-PA, TDI-positive asthma; TDI-NA, TDI-negative asthma; ES, exsmokers; NS, never smokers; SM, current smokers. $P$ values were obtained using the independent $t$-test or $\chi^{2}$-test and were considered significant when less than 0.05 .

TABLE 2: Comparison of GSTM1 and GSTT1 genotype frequencies according to the response of the TDI challenge.

\begin{tabular}{|c|c|c|c|}
\hline Genotype & TDI-PA (\%)/TDI-NA (\%) & OR $(95 \% \mathrm{CI})$ & $P$ value \\
\hline \multicolumn{4}{|l|}{ GSTM1 } \\
\hline Wild & $6(22.2) / 13(50.0)$ & 1.0 (reference) & \multirow{2}{*}{0.035} \\
\hline Null & $21(77.8) / 13(50.0)$ & $3.50(1.06-11.49)$ & \\
\hline \multicolumn{4}{|l|}{ GSTT1 } \\
\hline Wild & $11(40.7) / 15(57.7)$ & 1.0 (reference) & \multirow{2}{*}{0.217} \\
\hline Null & $16(59.3) / 11(42.3)$ & $1.98(0.66-5.91)$ & \\
\hline
\end{tabular}

TDI-PA: TDI challenge positive asthma and TDI-NA: TDI challenge negative asthma. $P$ values were obtained using logistic regression analysis and were considered significant when less than 0.05 .

TABle 3: Combined analysis of GSTM1 and GSTT1 mutant frequencies according to the response of the TDI challenge.

\begin{tabular}{lccc}
\hline Genotypes & TDI-PA (\%)/TDI-NA (\%) & OR (95\% CI) & $P$ value \\
\hline GSTM1 wild/GSTT1 wild & $3(11.1) / 6(23.0)$ & 1.0 (reference) & - \\
GSTM1 null/GSTT1 wild & $8(29.6) / 9(34.6)$ & $1.77(0.33-9.55)$ & 0.50 \\
GSTM1 wild/GSTT1 null & $3(11.1) / 7(26.9)$ & $0.85(0.12-5.94)$ & 0.87 \\
GSTM1 null/GSTT1 null & $13(48.2) / 4(15.3)$ & $6.50(1.09-38.63)$ & 0.04 \\
\hline
\end{tabular}

TDI-PA: TDI challenge positive asthma and TDI-NA: TDI challenge negative asthma. $P$ values were obtained using logistic regression analysis and were considered significant when less than 0.05 .

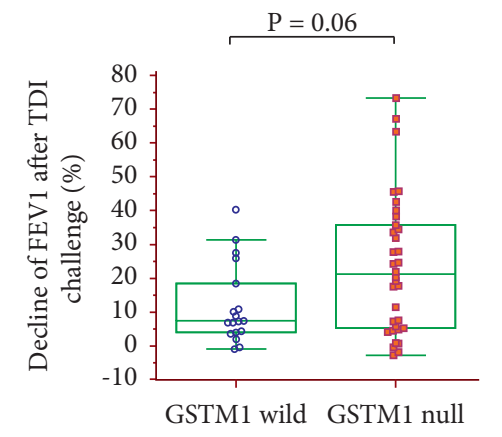

(a)

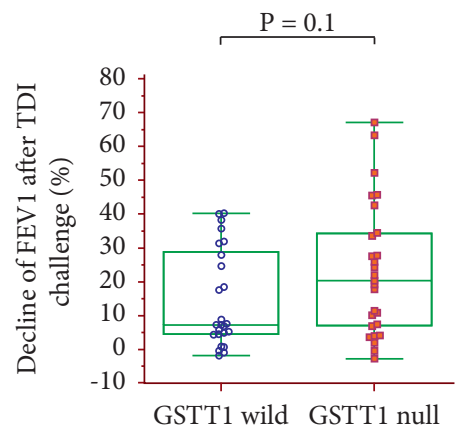

(b)

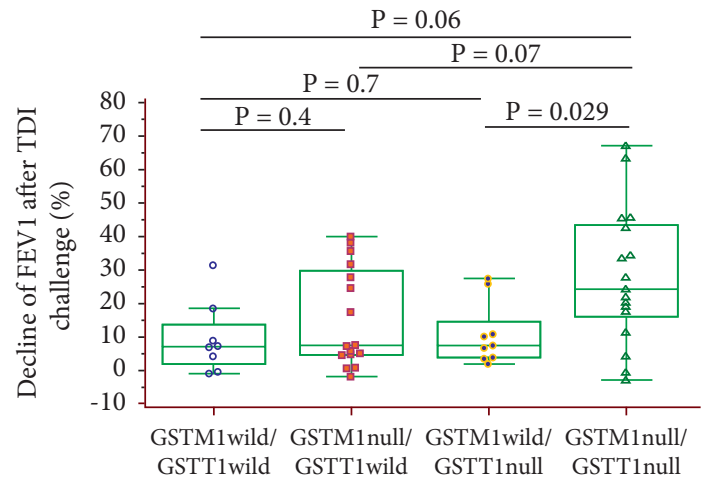

(c)

FIGURE 2: Decline in FEV1 (\%) after the TDI challenge according to the GSTM1 and GSTT1 genotypes. Comparison of dFEV1-TDI between the GSTM1 null and wild-type genotypes (a), between the GSTT1 null and wild-type genotypes (b), and among the combined GSTM1 and GSTT1 genotypes (c). 


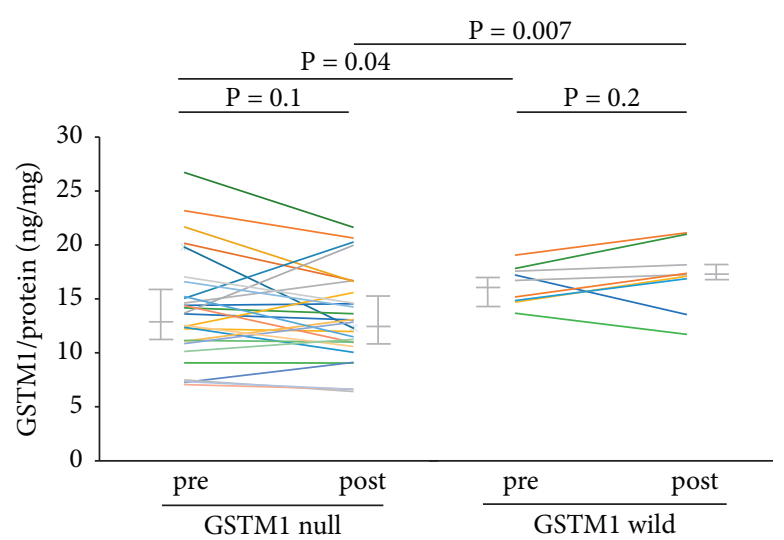

(a)

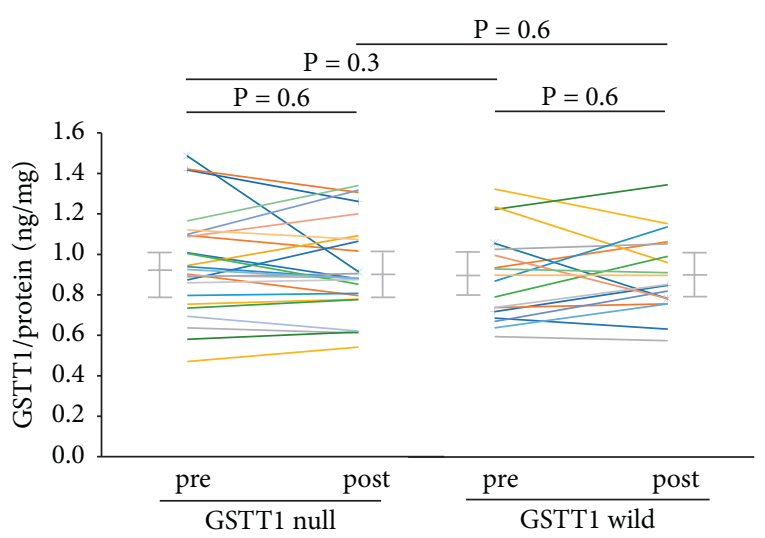

(b)

FIGURE 3: Plasma GSTM1 and GSTT1 protein levels according to genotype. The plasma GSTM1 protein concentrations before and after the TDI challenge in individuals with the null $(n=31)$ or wild-type $(n=12)$ GSTM1 genotype (a). The plasma GSTT1 protein concentrations before and after the TDI challenge in individuals with the null $(n=25)$ or wild-type $(n=18)$ GSTT1 genotypes (b).

function can trigger asthma onset via increased oxidative stress [34]. GST polymorphisms modulate the susceptibility to asthma. In a recent meta-analysis of 41 case-control studies, the pooled results showed a significant association of asthma with both the GSTM1 $(\mathrm{OR}=1.21)$ and GSTT1 $(\mathrm{OR}=1.61)$ genotypes [26]. Considering these data and ours, the dominant responding GST genotypes may depend on the exposure agent.

Genetic effects of the GST family on TDI-OA have been reported in several ethnic populations. In a study of Caucasians, the GSTM1 null genotype was associated with a 1.89-fold higher risk of diisocyanate-induced asthma $(n=109)$ compared with TDI exposure in the absence of asthma $(n=73)$, while the frequency of the GSTT1 null genotype did not differ between the two groups [27].These findings are in line with our finding that the GSTM1 null genotype was associated with an approximately three-fold increased risk of TDI-induced asthma, while the GSTT1 null genotype had an insignificant association. In our study, the frequencies of the GSTM1 and GSTT1 null genotypes were $77.8 \%$ and $59.3 \%$, respectively, which were greater than those reported in a Caucasian study (54.1\% and 11.9\%) [27]. This discrepancy might be attributed to racial differences. The GSTM1 and GSTT1 null genotype frequencies in the TDI-NA group in our study were $50 \%$ and $42.3 \%$, which are similar to those reported in 1,051 Korean males $(53.8 \%$ and $54.3 \%$, respectively) [35]. A study of workers with SICconfirmed diisocyanate-induced asthma $(n=95)$ revealed that the GSTM1 null and GSTP1 rs762803 genotypes were risk variants [36]. In another study of 92 TDI-OA patients exposed to TDI for 10 or more years, the frequency of the GSTP1 Val/Val genotype was associated with moderate-tosevere airway hyper-responsiveness to methacholine compared with subjects with normal or mild hyper-responsiveness [28]. Thus, of the GST family members, GSTM1 appears to be most frequently associated with diisocyanateinduced asthma.

Because GSTs have overlapping effects, they act synergistically as antioxidants. Such an effect was most marked for the combination of the GSTM1 null and GSTM3 AA genotypes, which was strongly associated with a late reaction in the bronchial provocation test in Caucasians. The genotype combinations GSTT1 * GSTP1 rs762803 and GSTM1 * EPHX1 rs2854450 were also associated with diisocyanate-induced asthma [36]. Furthermore, our study first demonstrated that the GSTM1/GSTT1 null genotype conferred a 6.5-fold increased risk of TDI-OA compared with the GSTM1/GSTT1 wild genotype. This suggests a possible additive contribution of GSTT1 to GSTM1 null genotypes in the development of TDI-OA. The frequency of homozygous deletion of GSTM1 and GSTT1 in our subjects $(n=17 / 53$; $32 \%)$ was similar to that in a large Korean population (29.1\%) and higher than that in a Caucasian population (7.5\%) [35]. dFEV1-TDI was highest in subjects with GSTM1/GSTT1 null among the four genotypes, with a significant difference compared with the GSTM1 wild/ GSTT1 null genotype. FEV1-TDI tended to be higher in subjects with the GSTM1 null than in wild-type genotypes. This suggests that GSTM1 and GSTT1 have a synergistically protective effect.

To validate the genetic effects of GSTM1 and GSTT1, the plasma GSTM1 and GSTT1 protein levels were measured. The GSTM1 protein concentration was significantly downregulated in GSTM1 null compared with wild-type patients, while the GSTT1 protein concentration did not differ between the genotypes, confirming that GSTM1 null patients are more susceptible to TDI-OA than GSTT1 null patients. Interestingly, the plasma GSTM1 and GSTT1 protein concentrations did not change after the TDI challenge regardless of the genotype. There might be delayed induction of these proteins. Because plasma GSTM1 and GSTT1 are produced mainly in the liver [37], a change in hepatic enzyme activity in response to inhalation of TDI might occur later in the circulation. The delayed response of GST proteins has been well demonstrated in diet studies. A 7 -day diet of cruciferous vegetables significantly increased the serum GSTM1 concentration [38]. In that study, the GSTM1 protein concentration was significantly higher on 
day 7 than on day 6 of the diet, suggesting that the response to the diet reached a steady state after 1 week. This may explain why the GSTM1 and GSTT1 levels did not change immediately after the TDI challenge. A long-term workplace exposure study may be needed to evaluate the different in vivo responses of GST family members according to genotypes.

Our study has several limitations. First, normal controls and non-TDI-exposed asthmatics were not included. The specificity of GSTM1 null genotypes to TDI-OA should be analyzed by comparisons with asthmatics with other types of occupational exposure. Second, other isoforms of GSTM and GSTT were not evaluated. Human cytosolic GSTs consist of five main classes (alpha, mu, pi, theta, and zeta) [18], and the human GSTM family consists of five different isotypes (GSTM1 to GSTM5) [19], while the GSTT family includes GSTT1, GSTT2, and GSTT2B [20].These other GST family members compensate for the absence of a functional GSTM1 enzyme [39]. Finally, we did not have sufficient statistical power to evaluate genotype $\times$ TDI interactions. When estimating the sample size for this study, we would have $83.67 \%$ power with a sample size of 64 . A post-hoc calculation based on our results indicates that our power was low ( $46.53 \%$ for overall effects). Therefore, it is possible that the significant results may also be explained by chance. However, our study was strengthened by the enrollment of subjects with correct diagnoses. TDI-OA is often diagnosed based on a history of work exposure to causative agents and the presence of asthma symptoms. However, this approach is not always satisfactory for diagnosing OA.29 Instead, we diagnosed TDI-PA by SIC using a TDI solution, which is considered the standard reference test [30].

\section{Conclusion}

The associations of the genotypes of the GSTM1 and GSTT1 genes with TDI-OA were evaluated in 26 TDI-PA and 27 TDI-NA individuals. The GSTM1 null genotype was more frequent in the TDI-PA group. In the combination analysis, the frequency of the GSTM1/GSTT1 null genotype was higher in the TDI-PA group than in the TDI-NA group. The plasma GSTM1 protein concentration was significantly lower in GSTM1 null than in wild-type individuals after the TDI challenge, while the GSTT1 protein concentration did not change in GSTT1 null and wild-type individuals after the TDI challenge. Thus, the GSTM1 null genotype may cause susceptibility to TDI-PA, and this effect may be synergistically enhanced by the GSTT1 null genotype. These data suggest that the GSTM1 and GSTT1 genotypes are useful diagnostic markers for TDI-OA.

$\begin{array}{ll}\text { Abbreviations } \\ \text { TDI: } & \text { Toluene diisocyanate } \\ \text { OA: } & \text { Occupational asthma } \\ \text { GSTM1: } & \text { Glutathione S-transferase Mu } 1 \\ \text { GSTT1: } & \text { Glutathione S-transferase Theta } 1 .\end{array}$

\section{Data Availability}

The data used to support the findings of this study are available from the corresponding author upon request.

\section{Additional Points}

(1) The GSTM1 null genotype may confer susceptibility to TDI-OA. (2) The genetic effect of the GSTM1 null genotype may be enhanced synergistically by the GSTT1 null genotype. (3) The GSTM1 and GSTT1 genotypes may be useful diagnostic markers for TDI-OA.

\section{Ethical Approval}

The study was conducted with the permission of the Ethics Committee of Soonchunhyang University.

\section{Consent}

Informed written consent for study participation and sample donation was obtained from each subject.

\section{Conflicts of Interest}

The authors declare that they have no conflicts of interest.

\section{Authors' Contributions}

Jong-Uk Lee and Ji-Yeon Jeong contributed equally to this work.

\section{Acknowledgments}

This study was supported by the Basic Science Research Program through the National Research Foundation of Korea (NRF) funded by the Ministry of Education (2020R1I1A1A01067088), the Korean Society for Environmental Asthma and Lung Diseases grant to Jong-Uk Lee, and a Soonchunhynag University grant to Jong Sook Park. The biospecimens and data used for this study were provided by the Biobank of Soonchunhyang University Bucheon Hospital, a member of the Korea Biobank Network (KBN4_A06).

\section{Supplementary Materials}

Study Subjects. Buffy coats and plasma were obtained from a biobank at Soonchunhyang University Hospital, Bucheon, Korea. Additionally, this study used fully anonymized data collected previously as part of the biobank at Soonchunhyang University Bucheon Hospital. (Supplementary Materials)

\section{References}

[1] J.-L. Malo and M. Chan-Yeung, "Occupational asthma," The Journal of Allergy and Clinical Immunology, vol. 108, no. 3, pp. 317-328, 2001.

[2] S.-C. Kwon, J. Song, Y.-k. Kim, and G. M. Calvert, "Workrelated asthma in Korea-findings from the Korea work- 
related asthma surveillance (KOWAS) program, 2004-2009," Allergy, Asthma \& Immunology Research, vol. 7, no. 1, pp. 51-59, 2015.

[3] A.-S. Jang, I.-S. Choi, Y. I. Koh, J.-D. Moon, and K.-J. Lee, "Increase in airway hyperresponsiveness among workers exposed to methylene diphenyldiisocyanate compared to workers exposed to toluene diisocyanate at a petrochemical plant in Korea," American Journal of Industrial Medicine, vol. 37, no. 6, pp. 663-667, 2000.

[4] D. L. Pham, T. H. Trinh, G.-Y. Ban, S.-H. Kim, and H.-S. Park, "Epithelial folliculin is involved in airway inflammation in workers exposed to toluene diisocyanate," Experimental \& Molecular Medicine, vol. 49, no. 11, p. e395, 2017.

[5] Z. L. Lummus, A. V. Wisnewski, and D. I. Bernstein, "Pathogenesis and disease mechanisms of occupational asthma," Immunology and Allergy Clinics of North America, vol. 31, no. 4, pp. 699-716, 2011.

[6] A. V. Wisnewski and C. A. Redlich, "Recent developments in diisocyanate asthma," Current Opinion in Allergy and Clinical Immunology, vol. 1, no. 2, pp. 169-175, 2001.

[7] Y. S. Shin, M. A. Kim, L.-D. Pham, and H.-S. Park, "Cells and mediators in diisocyanate-induced occupational asthma," Current Opinion in Allergy and Clinical Immunology, vol. 13, no. 2, pp. 125-131, 2013.

[8] J. Elms, P. N. Beckett, P. Griffin, and A. D. Curran, "Mechanisms of isocyanate sensitisation. an in vitro approach," Toxicology in Vitro, vol. 15, no. 6, pp. 631-634, 2001.

[9] P. K. Mishra, H. Panwar, A. Bhargava et al., "Isocyanates induces DNA damage, apoptosis, oxidative stress, and inflammation in cultured human lymphocytes," Journal of Biochemical and Molecular Toxicology, vol. 22, no. 6, pp. 429-440, 2008.

[10] P. K. Mishra, S. Khan, A. Bhargava et al., "Regulation of isocyanate-induced apoptosis, oxidative stress, and inflammation in cultured human neutrophils," Cell Biology and Toxicology, vol. 26, no. 3, pp. 279-291, 2010.

[11] P. K. Mishra, G. V. Raghuram, H. Panwar, D. Jain, H. Pandey, and K. K. Maudar, "Mitochondrial oxidative stress elicits chromosomal instability after exposure to isocyanates in human kidney epithelial cells," Free Radical Research, vol. 43, no. 8, pp. 718-728, 2009.

[12] H. Panwar, D. Jain, S. Khan et al., "Imbalance of mitochondrial-nuclear cross talk in isocyanate mediated pulmonary endothelial cell dysfunction," Redox Biology, vol. 1, no. 1, pp. 163-171, 2013.

[13] I. Rahman, S. K. Biswas, and A. Kode, "Oxidant and antioxidant balance in the airways and airway diseases," $E$ ropean Journal of Pharmacology, vol. 533, no. 1-3, pp. 222-239, 2006.

[14] A. J. Dozor, "The role of oxidative stress in the pathogenesis and treatment of asthma," Annals of the New York Academy of Sciences, vol. 1203, no. 1, pp. 133-137, 2010.

[15] E. Birben, U. M. Sahiner, C. Sackesen, S. Erzurum, and O. Kalayci, "Oxidative stress and antioxidant defense," World Allergy Organization Journal, vol. 5, no. 1, pp. 9-19, 2012.

[16] J. E. Ladner, J. F. Parsons, C. L. Rife, G. L. Gilliland, and R. N. Armstrong, "Parallel evolutionary pathways for glutathione transferases: structure and mechanism of the mitochondrial class kappa enzyme rGSTK1-1," Biochemistry, vol. 43, no. 2, pp. 352-361, 2004.

[17] R. Udomsinprasert, S. Pongjaroenkit, J. Wongsantichon et al., "Identification, characterization and structure of a new delta class glutathione transferase isoenzyme," Biochemical Journal, vol. 388, no. Pt 3, pp. 763-771, 2005.
[18] A. Oakley, "Glutathione transferases: a structural perspective," Drug Metabolism Reviews, vol. 43, no. 2, pp. 138-151, 2011.

[19] W. R. Pearson, W. R. Vorachek, S. J. Xu et al., "Identification of class-mu glutathione transferase genes GSTM1-GSTM5 on human chromosome 1p13," The American Journal of Human Genetics, vol. 53, no. 1, pp. 220-233, 1993.

[20] A. Hollman, P. Tchounwou, and H.-C. Huang, "The association between gene-environment interactions and diseases involving the human GST superfamily with SNP variants," International Journal of Environmental Research and Public Health, vol. 13, no. 4, p. 379, 2016.

[21] Y.-J. Zhou, B.-L. Zhao, Z. Qian, Y. Xu, and Y.-Q. Ding, "Association of Glutathione S-Transferase M1 null genotype with inflammatory bowel diseases," Medicine, vol. 98, no. 44, Article ID e17722, 2019.

[22] S. Nath, S. Das, A. Bhowmik, S. K. Ghosh, and Y. Choudhury, "The GSTM1 and GSTT1 null genotypes increase the risk for type 2 diabetes mellitus and the subsequent development of diabetic complications: a meta-analysis," Current Diabetes Reviews, vol. 15, no. 1, pp. 31-43, 2019.

[23] M. Yang, J. Zhao, L. Xing, and L. Shi, "Association between GSTM1 null genotype and coronary artery disease risk: a meta-analysis," Medical Science Monitor: International Medical Journal of Experimental and Clinical Research, vol. 20, pp. 1550-1555, 2014.

[24] P. J. Castaldi, M. H. Cho, M. Cohn et al., "The COPD genetic association compendium: a comprehensive online database of COPD genetic associations," Human Molecular Genetics, vol. 19, no. 3, pp. 526-534, 2009.

[25] C. Carlsten, G. S. Sagoo, A. J. Frodsham, W. Burke, and J. P. T. Higgins, "Glutathione S-transferase M1 (GSTM1) polymorphisms and lung cancer: a literature-based systematic HuGE review and meta-analysis," American Journal of Epidemiology, vol. 167, no. 7, pp. 759-774, 2008.

[26] X. Su, Y. Ren, M. Li, L. Kong, and J. Kang, "Association of glutathione S-transferase M1 and T1 genotypes with asthma," Medicine, vol. 99, no. 34, Article ID e21732, 2020.

[27] P. Piirilä, H. Wikman, R. Luukkonen et al., "Glutathione S-transferase genotypes and allergic responses to diisocyanate exposure," Pharmacogenetics, vol. 11, no. 5, pp. 437-445, 2001.

[28] C. E. Mapp, A. A. Fryer, N. D. Marzo et al., "Glutathione S-transferase GSTP1 is a susceptibility gene for occupational asthma induced by isocyanates," The Journal of Allergy and Clinical Immunology, vol. 109, no. 5, pp. 867-872, 2002.

[29] J.-L. Malo, H. Ghezzo, J. L'archevêque, F. Lagier, B. Perrin, and A. cartier, "Is the clinical history a satisfactory means of diagnosing occupational asthma?" American Review of Respiratory Disease, vol. 143, no. 3, pp. 528-532, 1991.

[30] J. Beach, B. H. Rowe, S. Blitz et al., "Diagnosis and management of work-related asthma: summary," Chest, vol. 134, no. 3, pp. 1S-41S, 2008.

[31] H. S. Park, Y. S. Cho, J. N. Park et al., "Significant changes of bronchial responsiveness to methacholine after early asthmatic reaction to toluene diisocyanate (TDI) in a TDI-sensitive asthmatic worker," Journal of Korean Medical Science, vol. 5, no. 4, pp. 185-188, 1990.

[32] J. D. Hayes and R. C. Strange, "Glutathione S-transferase polymorphisms and their biological consequences," Pharmacology, vol. 61, no. 3, pp. 154-166, 2000.

[33] F. Hollins, A. Sutcliffe, E. Gomez et al., "Airway smooth muscle NOX4 is upregulated and modulates ROS generation in COPD," Respiratory Research, vol. 17, no. 1, p. 84, 2016. 
[34] S. A. A. Comhair and S. C. Erzurum, "Redox control of asthma: molecular mechanisms and therapeutic opportunities," Antioxidants and Redox Signaling, vol. 12, no. 1, pp. 93-124, 2010.

[35] H.-J. Cho, S.-Y. Lee, C.-S. Ki, and J.-W. Kim, "GSTM1, GSTT1 and GSTP1 polymorphisms in the Korean population," Journal of Korean Medical Science, vol. 20, no. 6, pp. 10891092, 2005.

[36] B. Yucesoy, V. J. Johnson, Z. L. Lummus et al., "Genetic variants in antioxidant genes are associated with diisocyanateinduced asthma," Toxicological Sciences, vol. 129, no. 1, pp. 166-173, 2012.

[37] C. S. Kang, S. S. Hong, J. S. Kim, and E. R. Kim, "Glutathione S-transferase polymorphism of neonatal hyperbilirubinemia in Korean neonates," Korean Journal of Pediatrics, vol. 51, no. 3, pp. 262-266, 2008.

[38] J. W. Lampe, C. Chen, S. Li et al., "Modulation of human glutathione S-transferases by botanically defined vegetable diets," Cancer Epidemiology, Biomarkers \& Prevention: A Publication of the American Association for Cancer Research, Cosponsored by the American Society of Preventive Oncology, vol. 9, no. 8, pp. 787-793, 2000.

[39] P. Bhattacharjee, S. Paul, M. Banerjee et al., "Functional compensation of glutathione S-transferase M1 (GSTM1) null by another GST superfamily member, GSTM2," Scientific Reports, vol. 3, no. 1, p. 2704, 2013. 